\title{
Correction to: Machine learning classifiers can predict Gleason pattern 4 prostate cancer with greater accuracy than experienced radiologists
}

\author{
Michela Antonelli ${ }^{1,2} \cdot$ Edward W. Johnston ${ }^{3} \cdot$ Nikolaos Dikaios $^{3} \cdot$ King K. Cheung $^{3} \cdot$ Harbir S. Sidhu $^{3} \cdot$ \\ Mrishta B. Appayya ${ }^{3}$. Francesco Giganti ${ }^{4,5}$. Lucy A. M. Simmons ${ }^{5}$. Alex Freeman ${ }^{6}$. Clare Allen ${ }^{4} \cdot$ Hashim U. Ahmed $^{5}$. \\ David Atkinson $^{3}$ - Sebastien Ourselin ${ }^{2} \cdot$ Shonit Punwani ${ }^{3,4}$
}

Published online: 10 September 2019

(C) European Society of Radiology 2019

\section{Correction to: European Radiology (2019) 29:4754-4764}

https://doi.org/10.1007/s00330-019-06244-2

The original version of this article, published on 11 June 2019, unfortunately contained a mistake. The following correction has therefore been made in the original: In section "Multiparametric MRI review," the readers mentioned in the first sentence were partly incorrect. The correct wording is as follows: "Mp-MRI images were qualitatively assessed on an OsiriX workstation by three board-certified radiologists independently (readers SP, MA, and FG)."

Publisher's note Springer Nature remains neutral with regard to jurisdictional claims in published maps and institutional affiliations.

The online version of the original article can be found at https://doi.org/ 10.1007/s00330-019-06244-2

Shonit Punwani

shonit.punwani@gmail.com

1 Centre for Medical Image Computing, University College London, London, UK

2 School of Biomedical Engineering and Imaging Science, King's College London, London, UK

3 Centre for Medical Imaging, University College London, 2nd Floor Charles Bell House, 43-45 Foley Street, London W1W 7TS, UK

4 Department of Radiology, University College London Hospital, London, UK

5 Division of Surgery and Interventional Science, University College London, London, UK

6 Department of Pathology, University College London Hospital, London, UK 